\title{
Type III Secretion-Dependent and -Independent Phenotypes Caused by Ralstonia solanacearum in Arabidopsis Roots
}

\author{
Haibin Lu, ${ }^{1}$ Saul Lema A, ${ }^{1}$ Marc Planas-Marquès, ${ }^{1}$ Alejandro Alonso-Díaz, ${ }^{1}$ Marc Valls,,${ }^{1,2,+}$ and \\ Núria S. Coll' ${ }^{1, \dagger}$ \\ ${ }^{1}$ Centre for Research in Agricultural Genomics (CSIC-IRTA-UAB-UB), Bellaterra, Catalonia, Spain; and ${ }^{2}$ Genetics Department, \\ Universitat de Barcelona, Catalonia, Spain
}

Accepted 17 August 2017.

\begin{abstract}
The causal agent of bacterial wilt, Ralstonia solanacearum, is a soilborne pathogen that invades plants through their roots, traversing many tissue layers until it reaches the xylem, where it multiplies and causes plant collapse. The effects of $R$. solanacearum infection are devastating, and no effective approach to fight the disease is so far available. The early steps of infection, essential for colonization, as well as the early plant defense responses remain mostly unknown. Here, we have set up a simple, in vitro Arabidopsis thaliana-R. solanacearum pathosystem that has allowed us to identify three clear root phenotypes specifically associated to the early stages of infection: root-growth inhibition, root-hair formation, and root-tip cell death. Using this method, we have been able to differentiate, on Arabidopsis plants, the phenotypes caused by mutants in the key bacterial virulence regulators $h r p B$ and $h r p G$, which remained indistinguishable using the classical soil-drench inoculation pathogenicity assays. In addition, we have revealed the previously unknown involvement of auxins in the root rearrangements caused by $R$. solanacearum infection. Our system provides an easy-to-use, high-throughput tool to study $\boldsymbol{R}$. solanacearum aggressiveness. Furthermore, the observed phenotypes may allow the identification of bacterial virulence determinants and could even be used to screen for novel forms of early plant resistance to bacterial wilt.
\end{abstract}

The soilborne phytopathogen Ralstonia solanacearum is the causal agent of bacterial wilt, one of the most destructive bacterial crop diseases worldwide (Hayward 1991; Mansfield et al. 2012). Also referred to as the $R$. solanacearum species complex because of its wide phylogenetic diversity, this bacterium can cause disease on more than 200 plant species, including many important economic crops (Genin and Denny

Haibin Lu, Saul A. Lema, and Marc Planas-Marquès contributed equally to this work.

Current address for Haibin Lu: State Key Laboratory of Crop Stress Biology for Arid Areas and College of Agronomy, Northwest A\&F University, No.3 Taicheng Road, Yangling, Shaanxi 712100, China.

${ }^{\dagger}$ Corresponding authors: Marc Valls; E-mail: marcvalls@ub.edu and Núria S. Coll; E-mail: nuria.sanchez-coll@cragenomica.es

*The $\boldsymbol{e}$-Xtra logo stands for "electronic extra" and indicates that five supplementary figures and one supplementary table are published online.

@ 2018 The American Phytopathological Society
2012). R. solanacearum accesses the plant through the root and traverses many root layers until it reaches the xylem, where it profusely multiplies. From there, it spreads through the aerial part and causes wilting of the stem and leaves (Genin 2010).

Wilting symptoms caused by $R$. solanacearum are largely dependent on the presence of a functional type III secretion system (T3SS) (Boucher et al. 1985). The T3SS is a needle-like structure present in many pathogenic bacteria that allows secretion of virulence proteins - called effectors-into the host cells (Galán and Collmer 1999; Hueck 1998). In plant-associated bacteria, the genes responsible for the regulation and assembly of the T3SS are known as hypersensitive response and pathogenicity ( $h r p$ ) genes (Lindgren et. al. 1986). Transcription of the hrp genes and its related effectors is activated by $\operatorname{HrpB}$, the downstream regulator of a well-described regulatory cascade induced by contact with the plant cell wall (Brito et al. 2002). The cascade includes the membrane receptor PrhA, the signal transducer PrhI, and the transcriptional regulators PrhJ and HrpG (Brito et al. 2002). HrpG is downstream of PrhJ and directly controls HrpB expression (and thus, expression of the T3SS genes), but it also activates a number of HrpB-independent virulence determinants, such as genes for ethylene synthesis (Valls et al. 2006).

Since the establishment of the $R$. solanacearum pathosystem almost two decades ago, leaf wilting has been typically used as the major readout to study the Arabidopsis thaliana-R. solanacearum interactions (Deslandes et al. 1998). Soil drenching with a bacterial suspension, followed by leaf symptom evaluation over a time course, constitutes a solid measure to quantify the degree of resistance or susceptibility of the plant toward the pathogen. The disadvantages of this system are the uncontrolled influence of soil microbiota and its high variability due to infection stochasticity, as shown in potato (Cruz et al. 2014). In addition, leaf wilting is the last step of $R$. solanacearum infection and does not provide information about early steps of colonization. Furthermore, soil opacity hinders direct observation of any morphological changes associated to bacterial invasion of plant tissues.

The establishment of gnotobiotic assays in which $R$. solanacearum is inoculated on plants grown axenically has opened the door to studying the early steps of infection. $R$. solanacearum in vitro inoculation assays have been successfully established for tomato (Vasse et al. 1995), petunia (Zolobowska and Van Gijsegem 2006), and the model plants Medicago truncatula (Vailleau et al. 2007) and Arabidopsis thaliana (Digonnet et al. 2012). These studies have shed light on some common, as well as species-specific, root phenomena associated with $R$. solanacearum infection. Reduced primary root elongation after infection is a common feature observed in all species analyzed. Other common root phenotypes that appeared after 
infection were swelling of the root tip (in tomato, petunia, and M. truncatula), inhibition of lateral root growth (in petunia and Arabidopsis), and cell death (in M. truncatula and Arabidopsis). In petunia, $R$. solanacearum infection resulted as well in the formation of root lateral structures (Zolobowska and Van Gijsegem 2006). These structures resembled prematurely terminated lateral roots, were present both in resistant and susceptible lines, and were efficient colonization sites.

In vitro pathosystems have helped define the different stages of $R$. solanacearum infection. The bacterium was found to gain access into the tomato root through wound sites or natural openings such as emerging lateral roots (Saile et al. 1997; Vasse et al. 1995). In M. truncatula and Arabidopsis, the bacteria can also enter intact roots through the root apex (Digonnet et al. 2012; Vailleau et al. 2007). In petunia, it was shown that penetration occurs equally in resistant or susceptible plants (Zolobowska and Van Gijsegem 2006). The second stage of infection involves invasion of the root cortical area. In this stage, $R$. solanacearum quickly transverses the root cylinder centripetally via intercellular spaces, directed to the vasculature (Digonnet et al. 2012; Vasse et al. 1995). Massive cortical cell degeneration can be observed during this phase. The fact that cells not directly in contact with the bacteria also die led to a proposal that certain cell-wall fragments degraded by $R$. solanacearum may act as signals to induce plant programmed cell death (Digonnet et al. 2012). During the third stage of infection, $R$. solanaceraum enters into the vascular cylinder and colonizes the xylem. In Arabidopsis, it was shown that vascular invasion is promoted by collapse of two xylem pericycle cells (Digonnet et al. 2012). Once inside the xylem, bacteria start proliferating and moving between adjacent vessels by degrading the cell walls but remain confined in the xylem. In the last stage of infection, disease symptoms become apparent at the whole organism level, as the stem and leaves start wilting.

All these studies have significantly broadened our understanding of the root invasion process. However, the molecular mechanisms that control these phenotypes and their timing remain vastly unexplored. In addition, no clear correlation has been established between any of the observed phenotypes and the host's resistance or susceptibility to $R$. solanacearum. Here, we have set up a simple in vitro pathosystem to determine the impact of $R$. solanacearum on Arabidopsis root morphology at the first stages of infection.

\section{RESULTS}

\section{In vitro infection with $R$. solanacearum causes a triple phenotype on Arabidopsis roots.}

To analyze the impact of $R$. solanacearum infection on Arabidopsis root morphology, we established a simple in vitro inoculation assay. Sterile seeds were sown on Murashige Skoog (MS) media plates and were grown vertically for 7 days so that plant roots developed at the surface of the medium and could be easily inoculated and visualized. Plantlets were then inoculated $1 \mathrm{~cm}$ above the root tip with $5 \mu \mathrm{l}$ of a solution containing $R$. solanacearum. Infection with the wild-type GMI1000 strain caused root-growth arrest (Fig. 1A). To determine whether this effect depended on the inoculation point, we inoculated at the top, middle, and tip of the root. $R$. solanacearum causes root-growth inhibition regardless of the infection point (Supplementary Fig. S1). Hence, all experiments were performed inoculating $1 \mathrm{~cm}$ above the root tip. Interestingly, along with root-growth inhibition, we observed two additional root phenotypes caused by $R$. solanacearum infection, i.e., production of root hairs at the root-tip maturation zone (Fig. 1B) and cell death at

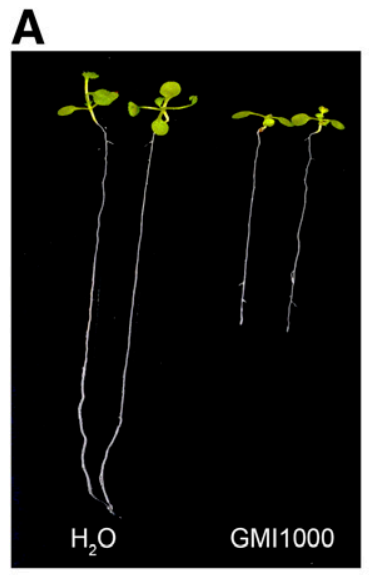

B

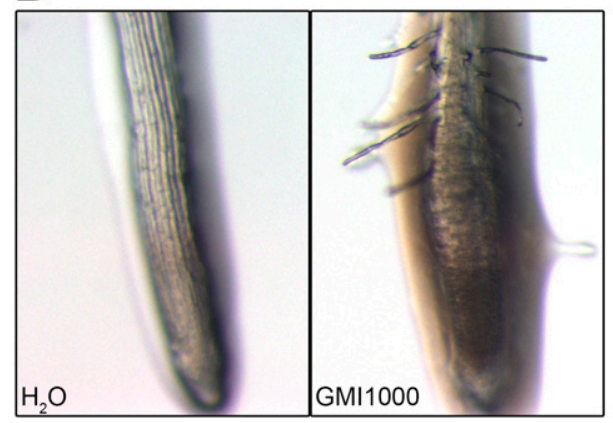

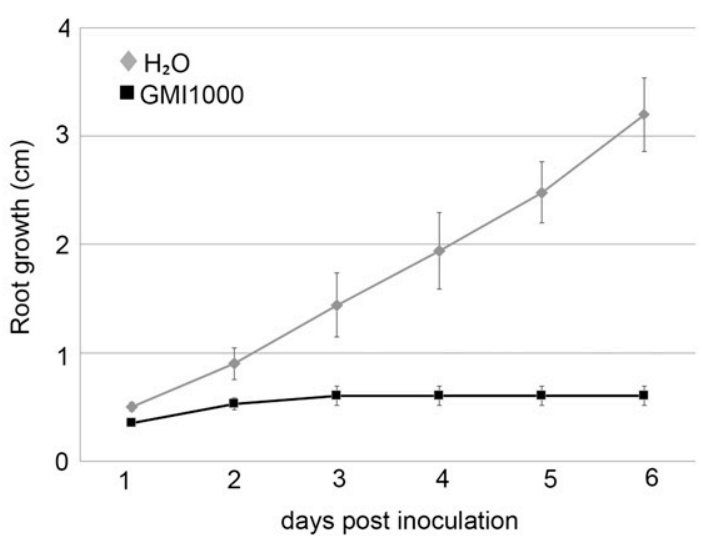

C

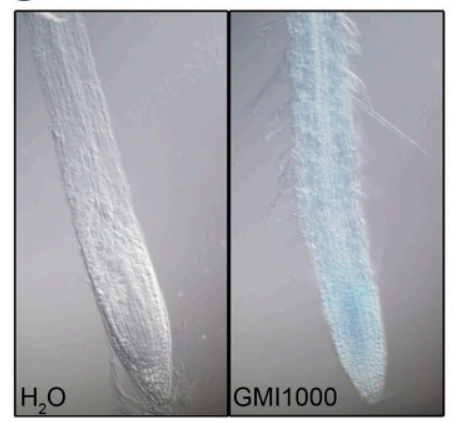

Fig. 1. Root phenotypes caused by Ralstonia solanacearum GMI1000 in vitro infection. Six-day-old Col-0 seedlings were inoculated with $5 \mu$ l of a GMI1000 solution or with water as a control. A, GMI1000 inhibition of root growth. The left panel shows stereoscope images of the plantlets under white light at 6 days postinoculation (dpi), and the right panel presents root length data at different times after infection. B, Root-hair formation on the root tip caused by GMI1000 infection. Root-tip pictures obtained, as before, at 6 dpi. C, Observation of cell death at root tips visualized by Evans blue staining. Representative Nomarski microscope pictures of stained roots obtained $6 \mathrm{dpi} ; 10$ to 15 plants were used in three independent experiments. 
the root tip. Cell death was visualized as either Evans blue (Fig. 1C) or propidium iodide staining (Supplementary Fig. S2), both of which are commonly used as cell-death markers as they are excluded from living cells by the plasma membrane (Curtis and Hays 2007; Gaff and Okong'O-gola 1971).

\section{R. solanacearum hrp mutants are altered in their capacity to cause the triple-root phenotype.}

With these three phenotypes in hand, we set out to identify their causative bacterial genetic determinants. For this, we analyzed the triple-root phenotypes on plants inoculated with $R$. solanacearum GMI1000 carrying mutations on the master regulators of virulence HrpG and HrpB. Bacteria bearing a disrupted $h r p G$ lost the ability to inhibit root growth but not those bearing disrupted $h r p B$ versions ( $h r p B$ and $h r p B \Omega$ ) (Fig. 2A).
Inoculation with the $\Delta h r p G$, in which the whole open reading frame had been deleted, and its complemented strain, $\Delta h r p G$ ( $h r p G$ ), confirmed the requirement of HrpG but not HrpB to induce the phenotypes. Similarly, bacterial strains disrupted in the membrane receptor $\operatorname{prh} A$, the signal transducer $\operatorname{prhI}$, and, to a lesser extent, the transcriptional regulator $p r h J$ were all strongly affected in their capacity to inhibit root growth (Fig. 3). This is logical, since all these mutants show decreased hrpG transcription (Brito et al. 2002). Since hrp mutants are all nonpathogenic (Boucher et al. 1985), the key role of HrpG in root inhibition compared with HrpB could be due to the fact that HrpG controls a larger number of bacterial virulence activities that have been proposed to be required for xylem colonization (Valls et al. 2006; Vasse et al. 2000). To check if root phenotypes correlated with bacterial colonization, 4-week-old
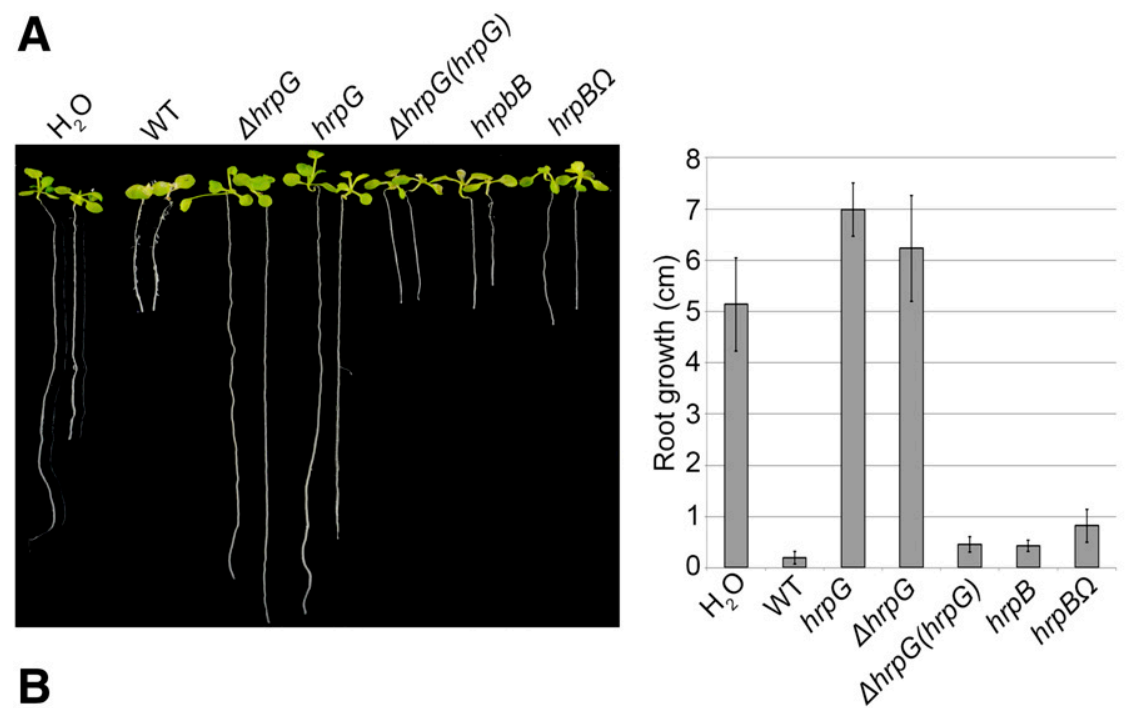

B

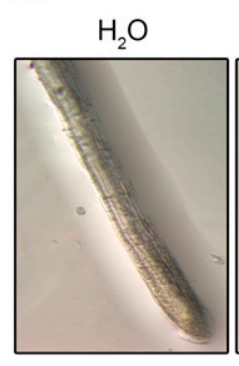

WT

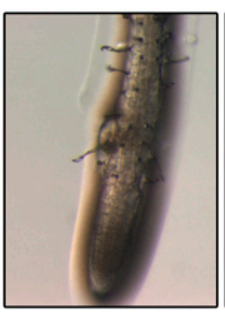

$h r p B$

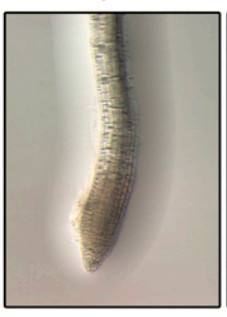

$h r p G$

$\Delta h r p G(h r p G)$

C

$\mathrm{H}_{2} \mathrm{O}$

WT

$h r p B$
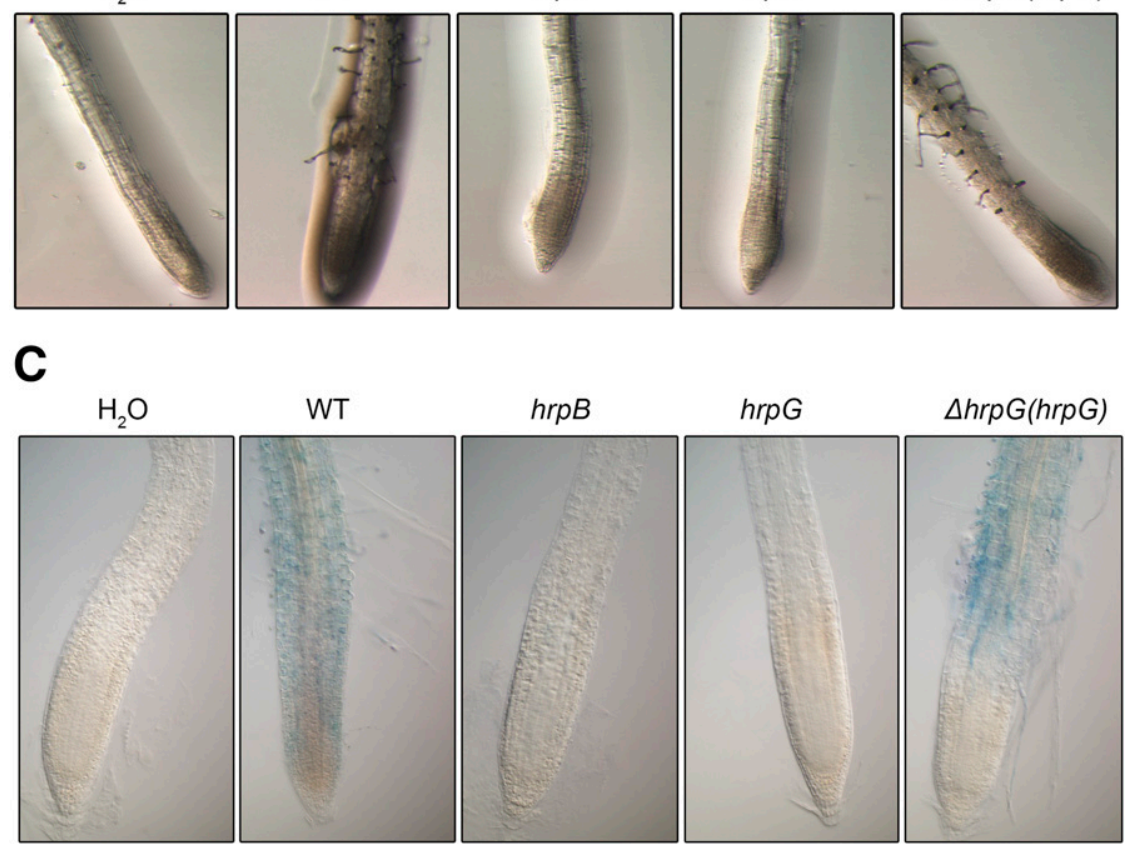

hrpG

$\Delta h r p G(h r p G)$

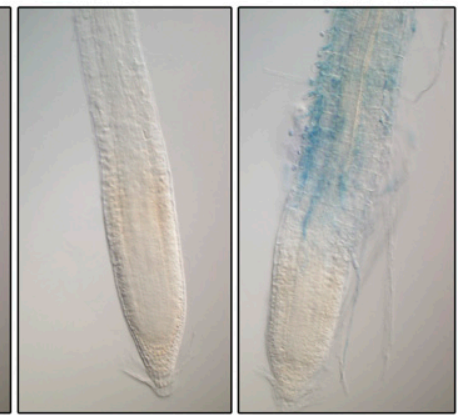

Fig. 2. HrpG is required for all the phenotypes caused by GMI1000, while HrpB is only essential for cell death and root-hair formation. Six-day-old Col-0 seedlings were inoculated with water (control) or with the following strains: GMI1000 wild type (WT), $\Delta h r p G$ (whole gene deletion), $h r p G$ (Tn5 transposon insertion), $\Delta h r p G$ (hrpG), $h r p B$ ( $\operatorname{Tn} 5$ transposon insertion), and $h r p B \Omega$ ( $\Omega$ cassette insertion). A, Mutations on HrpG but not on HrpB abolish growth inhibition. Left panel presents a picture taken at 9 days postinoculation (dpi), and the right panel presents root growth measurements at 9 dpi. B, Both $h r p G$ and $h r p B$ mutations abolish root-hair formation. Pictures were taken at 6 dpi. C, Neither the $h r p G$ nor $h r p B$ mutant cause root-tip cell death. Pictures of infected seedlings at 6 dpi stained with Evans blue. Each experiment was repeated at least three times, using five to ten plants. 
Arabidopsis Col-0 plants were inoculated with the wild-type $R$. solanacearum GMI1000 or its hrpB and hrpG deletion mutant counterparts. Bacterial loads were measured in aerial tissues of inoculated Arabidopsis plants 14 days after inoculation as colonyforming units (CFU) per gram of tissue. Supplementary Fig. S3 shows that the capacity to colonize Arabidopsis plants of hrpB is significantly higher than of $h r p G$ mutants. Thus, although hrp mutants had been already described to multiply in planta (Hanemian et al. 2013), HrpG seems to be more essential than $\mathrm{HrpB}$ for the bacterium to colonize the plant xylem and reach the aerial tissues.

Finally, we also observed that mutations in the $\operatorname{hrpB}$ and $h r p G$ regulators abolished root-hair formation and cell death caused by $R$. solanacearum on roots (Fig. 2B and C). In summary, we proved that root-hair production and cell-death induction are T3SS-dependent phenotypes. In contrast, root-growth

A

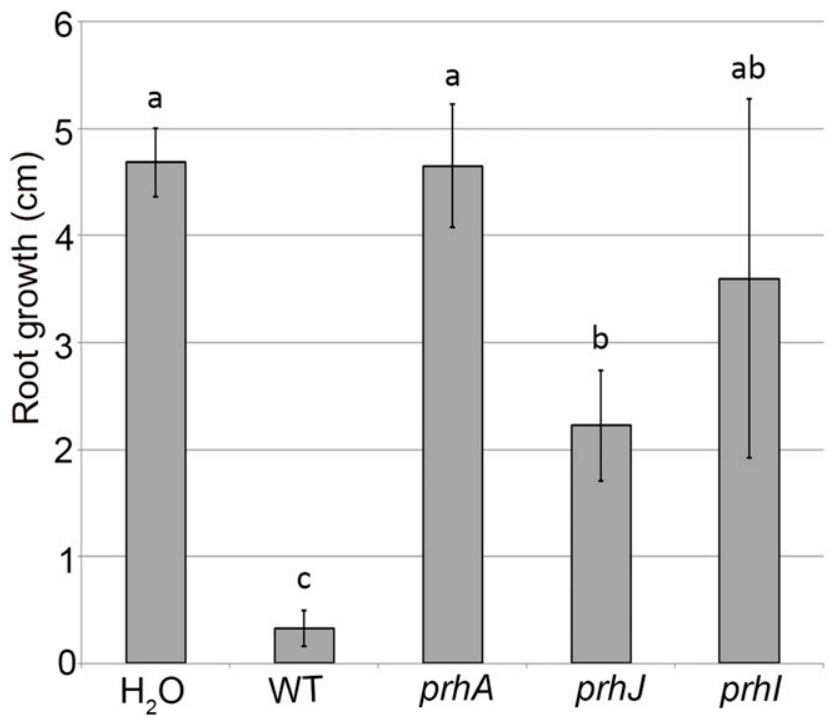

B

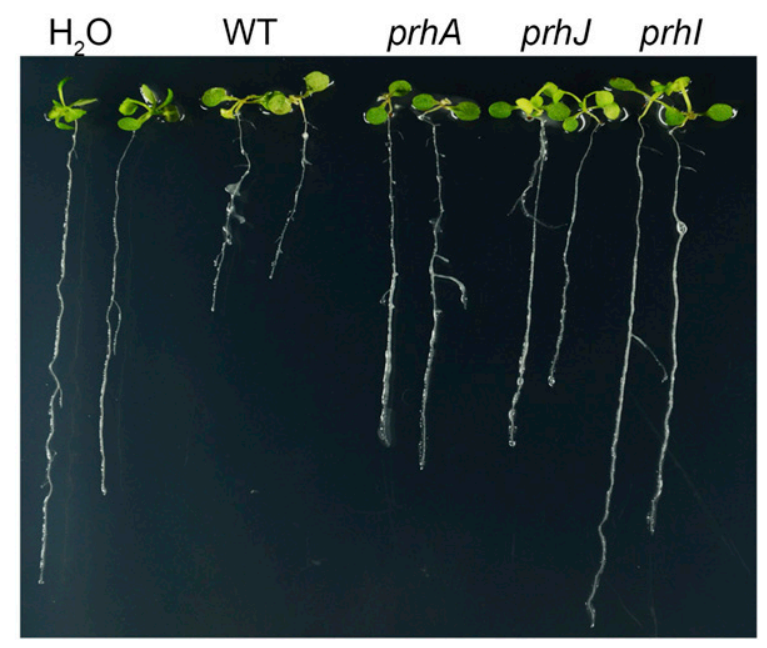

Fig. 3. Detection of plant signals is essential for GMI1000 to cause rootgrowth inhibition. Six-day-old Col-0 seedlings were inoculated with GMI1000 (WT), its derivative strains disrupted for components of the hrp signaling cascade or treated with water. A, Root growth was measured at 9 days postinoculation (dpi) and $\mathbf{B}$, pictures were taken at 9 dpi. Letters above bars indicate statistical significance; bars not sharing letters represent significant mean differences by one-way analysis of variance $(P<0.05$, $\alpha=0.05)$ with post hoc Scheffé $(\alpha=0.05)$. Five to seven plants were used in three independent experiments. inhibition, for which HrpG is required, does not depend on a functional T3SS.

\section{R. solanacearum strains unable to cause}

the triple-root phenotype are nonvirulent on Arabidopsis.

Our next goal was to determine whether the ability to cause the triple phenotype in Arabidopsis roots was conserved across different $R$. solanacearum strains and if there was a correlation to aggressiveness. For this, we inoculated in vitro-grown Arabidopsis Col-0 roots with $R$. solanacearum strains belonging to different phylotypes: our reference strain GMI1000 and strain Rd15 (phylotype I); CIP301 and CFBP2957 (phylotype IIA); NCPPB3987, UY031, and UW551 (phylotype IIB); and CMR15 (phylotype III). Interestingly, infection with phylotype IIA strains CIP301 and CFBP2957 resulted in root-growth inhibition (Fig. 4A), root-hair production (Fig. 4B) and cell death at the root tip (Fig. 4C), similar to what we observed with phylotype I and III strains. In contrast, phylotype IIB strains NCPPB3987, UY031, and UW551 did not cause growth inhibition nor roothair production or cell death on infected roots. Thus, different $R$. solanacearum strains vary in their ability to cause the tripleroot phenotype. To determine whether these phenotypes correlated with pathogenicity, we performed root infection assays on Arabidopsis plants grown on soil and recorded the appearance of wilting symptoms over time (Fig. 4D). Infection of wild-type Col-0 plants with the strains that were unable to cause the tripleroot phenotype (NCPPB3987, UY031, and UW551) did not result in wilting, which indicates a direct correlation between absence of root phenotypes in vitro and absence of symptoms in plants grown in soil. On the contrary, from all $R$. solanacearum strains causing the triple-root phenotype, only GMI1000, Rd15, and CMR15 resulted in plant wilting. As seen before for the $h r p G$ and $h r p B$ mutants, symptom scoring has limitations in evaluating slight $R$. solanacearum pathogenicity differences. Thus, we inoculated Arabidopsis plants with all studied bacterial strains and measured bacterial numbers in the aerial part 14 days postinoculation (dpi). The results, shown in Figure 4E, indicated that the two phylotype IIA strains (CIP301 and CFBP2957) that showed the triple phenotype but were not causing disease colonized the aerial part of the plants to higher numbers than the strains not causing the root responses. These results show that Arabidopsis root phenotypes partially correlate with the capacity of $R$. solanacearum to colonize Arabidopsis Col-0 plants; the strains that are not able to produce the triple-root phenotype are nonvirulent.

\section{$R$. solanacearum-triggered root-hair formation is mediated by plant auxins.}

To ascertain whether any of the phenotypes triggered by $R$. solanacearum infection were mediated by known plant defense regulators, we tested how different Arabidopsis mutants responded to the pathogen (Supplementary Fig. S4). Our results showed that reactive oxygen species (ROS) produced by the membrane NADPH oxidases AtRbohD and AtRbohF were not required for root-growth inhibition, root-hair production, or cell death in response to infection. Plants that were insensitive to jasmonic acid (jai3-1) or that could not synthetize it (dde2) or its conjugated form (jarl-1) showed root-growth inhibition, roothair production, and cell death similar to the wild type. Similarly, the sid2 mutant, defective in salicylic acid biosynthesis, and the ethylene insensitive mutant ein 2 responded with the same root morphologies as wild-type to $R$. solanacearum infection. On the contrary, the auxin insensitive mutants tirl and tirl/afb2 showed growth inhibition (Fig. 5A) and root-tip cell death (Fig. 5B) but were not able to produce root hairs in response to infection (Fig. 5C). This result indicates that root-hair production triggered by $R$. solanacearum infection requires auxin signaling. To monitor potential changes in auxin levels 
during infection, we analyzed expression of the auxin signaling reporter DR5rev::GFP in roots of infected versus control plants. As shown in Figure 5D, $R$. solanacearum inoculation induced a strong vascular green fluorescent protein (GFP) signal $48 \mathrm{~h}$ postinfection, suggesting that infection may result in increased auxin signaling levels in the vascular cylinder.

$R$. solanacearum encodes a HrpG-regulated ethylene-forming enzyme (efe) gene (Valls et al. 2006). To assess whether bacterial ethylene-mediated root-growth inhibition, we infected wild-type Arabidopsis with $R$. solanacearum GMI1000 wildtype strain or with the efe mutant. Supplementary Figure S5 shows that infection with the mutant resulted in root-growth inhibition, indicating that ethylene produced by the bacteria is not responsible for this phenotype. Bacterial ethylene was also not required for the root-hair formation phenotype, because infection with the efe mutant did not affect root-hair formation as expected if $\mathrm{HrpB}$, which does not activate the efe operon, controls this phenotype (Fig. 2B).

\section{Absence of the triple-root phenotype in Arabidopsis might reveal new sources of resistance to strain GMI1000.}

Next, we wanted to determine the degree of conservation of the correlation between absence of the triple phenotype and resistance to $R$. solanaceraum. For this, besides Col-0, we selected the accessions C24, Cvi-0, Ler-1, B1-1, and Rrs-7 from among the 20 proposed as representatives of the maximum variability of Arabidopsis (Delker et al. 2010). In addition, we included $\mathrm{Nd}-1$, known to be resistant to $R$. solanacearum (Deslandes et al. 1998), and Tou-A1-74, which does not show the triple phenotype (discussed below). Despite the differences in root length among accessions, the majority displayed the triple-root phenotype after inoculation with $R$. solanacearum
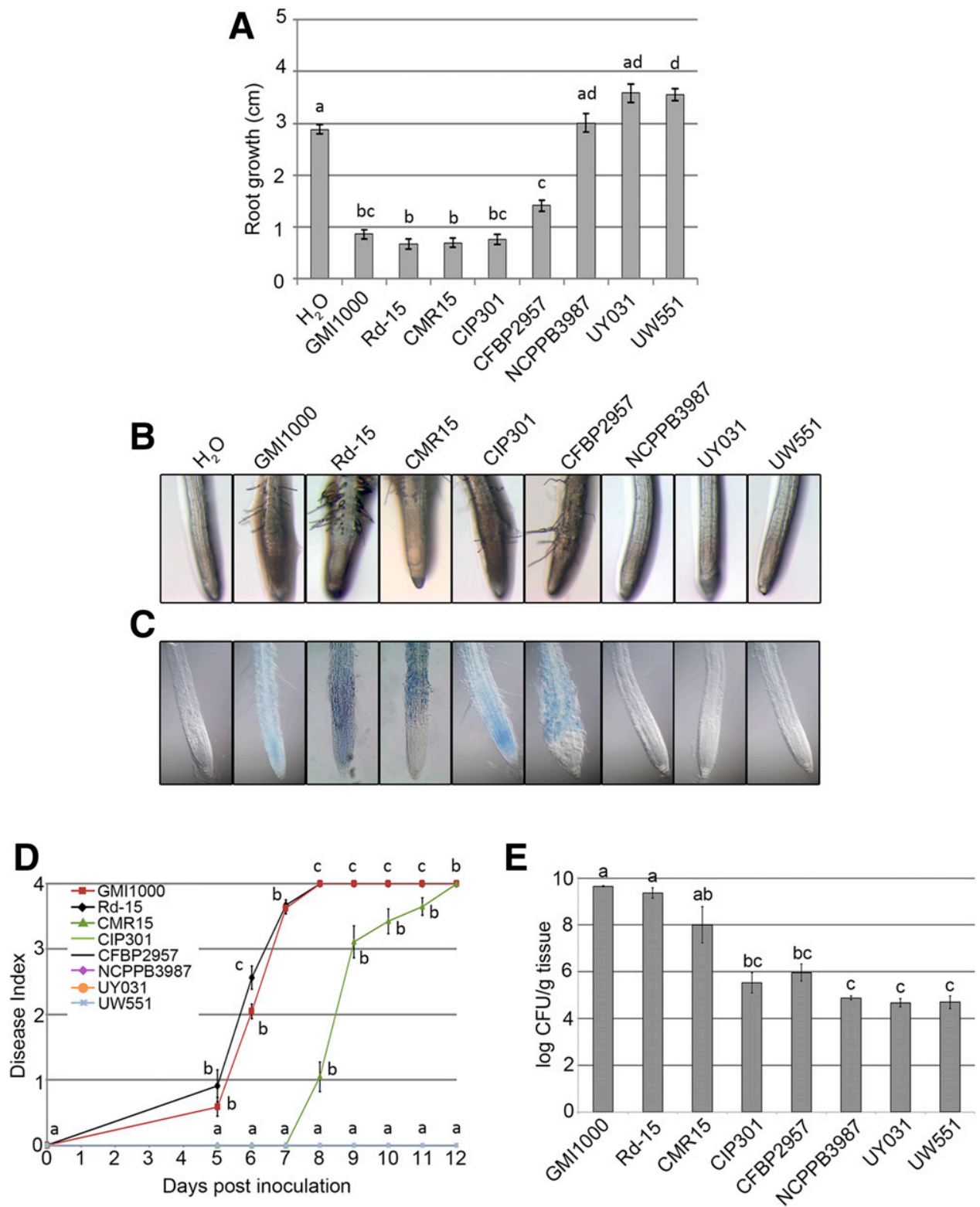

Fig. 4. The ability to cause root-growth inhibition, root-hair formation, and cell death varies across different Ralstonia solanacearum strains. Six-day-old Col-0 seedlings were inoculated with the indicated $R$. solanacearum wild-type strains or water. A, Root growth after infection at 6 days postinoculation (dpi). B, Pathogenicity assay. C, Bacterial multiplication in planta measured 14 days after inoculation. D, Root-hair formation at 6 dpi. E, Roots from infected seedlings, at $6 \mathrm{dpi}$, stained with Evans blue. For all graphs, letters indicate statistical significance; values not sharing letters represent significant mean differences by oneway analysis of variance $(P<0.05, \alpha=0.05)$ with post hoc Scheffé $(\alpha=0.05)$. In $\mathrm{B}$, the statistical test was applied separately for each timepoint. Each experiment was repeated at least three times using ten to 15 plants. 
(Fig. 6A, B, C). Only Rrs-7 and Tou-A1-74 did not show any of the three phenotypes in response to infection. To determine whether the presence or absence of the triple phenotype correlated to susceptibility to $R$. solanacearum GMI1000, we performed a pathogenicity assay using these accessions (Fig. 6D). Interestingly, Rrs-7 but not Tou-A1-74 was resistant to $R$. solanacearum, indicating that absence of the root phenotypes could be used to identify some sources of resistance to the pathogen. Resistance to $R$. solanacearum was not found in random accessions showing

A
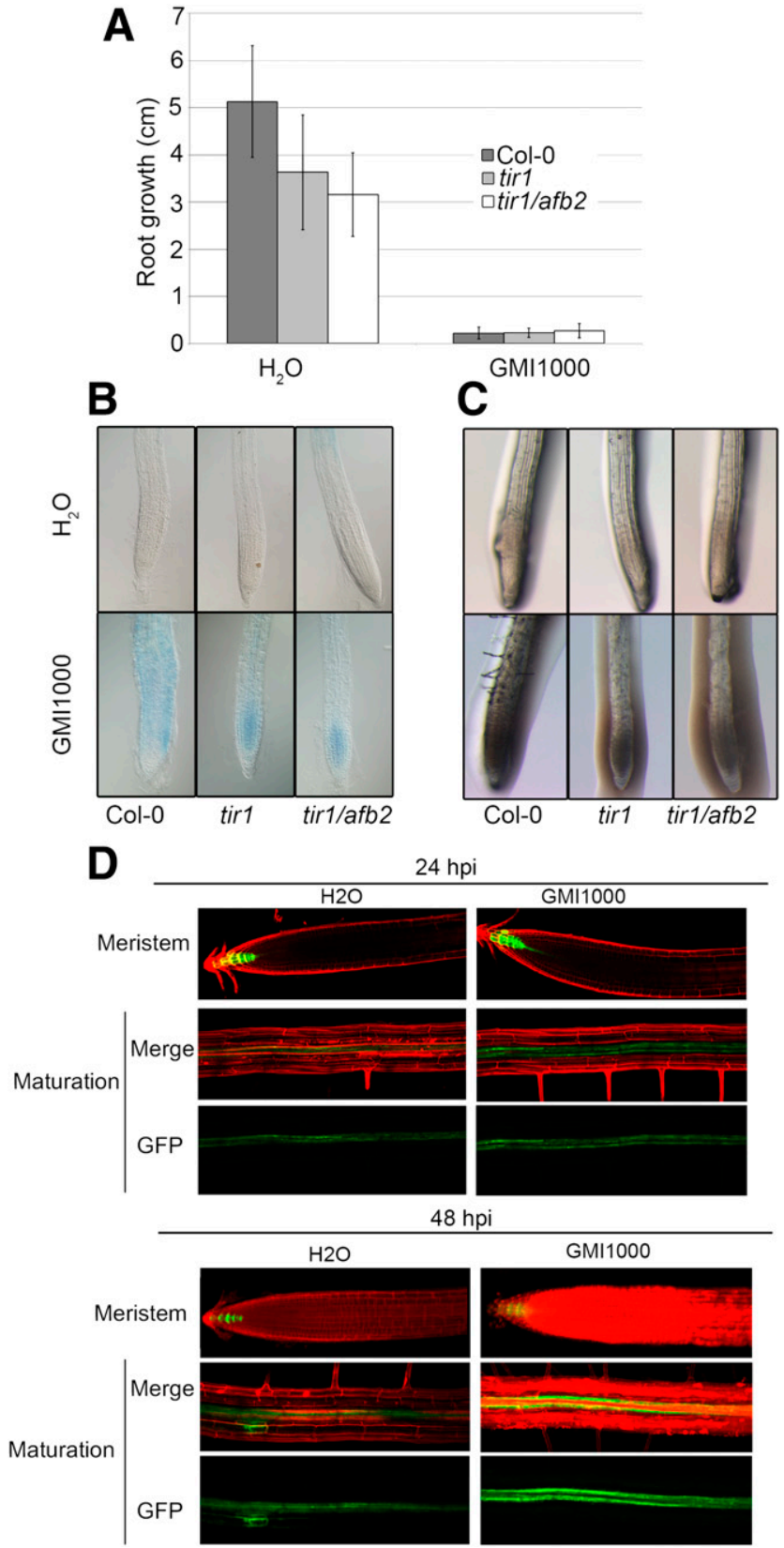

Fig. 5. Auxin signaling is required for Ralstonia solanacearum-triggered root-hair formation in Arabidopsis but not for root-growth inhibition and cell death. Six-day-old Col-0, tirl, and tirl/afb2 seedlings were inoculated with $R$. solanacearum GMI1000 or water and, at 6 days postinoculation, A, root growth was measured $\mathbf{B}$, root-hair formation was evaluated, and C, roots from infected seedlings were stained with Evans blue. D, Expression of the auxin signaling marker DR5 was analyzed under the confocal microscope in roots of transgenic Col-0 DR5rev::GFP plants infected with $R$. solanacearum GMI1000 or water at 24 and $48 \mathrm{~h}$ after inoculation. Representative pictures of both the meristem area and maturation zone are shown. Six to ten plants were used in three different experimental replicates. the triple-root phenotype, which, however, did not correlate with susceptibility, since the resistant accessions Nd-1 (Deslandes et al. 1998) and Bl-1 reacted with root-growth inhibition, roothair production, and cell death after infection (Fig. 6D).

\section{DISCUSSION}

Plant host root phenotypes appear as early symptoms of colonization by $R$. solanacearum.

The use of in vitro pathosystems to study the interactions between the vascular pathogen $R$. solanacearum and some of its plant hosts has emerged as a very powerful technique to understand the early stages of infection (Digonnet et al. 2012; Turner et al. 2009; Vailleau et al. 2007; Vasse et al. 1995, 2000; Zolobowska and Van Gijsegem 2006). In this work, we have used in vitro-grown Arabidopsis as the model host to deepen our knowledge on the first steps of $R$. solanacearum root invasion. In vitro infection has several advantages: i) it reveals easily screenable root phenotypes associated with the infection that would remain hidden when using the soil-drench inoculation; ii) it facilitates microscopy studies to determine the penetration point and the infection itinerary through the root cell layers; and iii) it is a useful tool to study the genetic determinants controlling both $R$. solanacearum virulence and host defense.

A very detailed microscopic analysis of the gnotobiotic Arabidopsis $-R$. solanacearum interaction has been recently published (Digonnet et al. 2012). This study revealed the path followed by $R$. solanacearum through Arabidopsis roots, highlighting the sites of bacterial multiplication and the specific cellwall barriers degraded by the bacterium. Moving forward this knowledge, our data defines a set of root phenotypes associated to infection that can be correlated to bacterial aggressiveness and plant resistance and are genetically amenable, both from the bacterial and the plant side.

In our system, infection of intact roots with a droplet of $R$. solanacearum resulted in root-growth inhibition, roothair production, and cell death. Root-growth inhibition or delayed elongation has been previously observed as a result of $R$. solanacearum infection, when using gnotobiotic systems (Digonnet et al. 2012; Turner et al. 2009; Vailleau et al. 2007; Vasse et al. 1995; Zolobowska and Van Gijsegem 2006). One could hypothesize that root-growth inhibition is the direct cause of the massive cell death observed after infection in the root cortex of Arabidopsis (Digonnet et al. 2012; this work) or other species (Turner et al. 2009; Vasse et al. 1995). However, this does not seem to be the case, since a $h r p B$ mutant strain causes rootgrowth inhibition in the absence of cell death. Considering this, root-growth inhibition would rather reflect xylem colonization, which takes place both for wild-type $R$. solanacearum GMI1000 and the $h r p B$ mutant. In agreement with this interpretation, the $h r p G$ mutant, which has an extremely reduced capacity to invade the xylem (Vasse et al. 1995, 2000), does not cause root-growth inhibition after infection. This further highlights the proposed role of $\mathrm{HrpG}$ as a central regulator controlling still-unknown activities essential for the bacterium to reach and multiply in the plant xylem (Valls et al. 2006; Vasse et al. 2000). These activities are likely encoded in genes regulated by HrpG independently of $\mathrm{HrpB}$, as the latter is able to colonize the xylem. Among the 184 genes specifically regulated by $\mathrm{HrpG}$, an obvious candidate responsible for the root-growth inhibition is the gene controlling bacterial production of the phytohormone ethylene. However, we found the bacterial mutant defective in this gene still inhibited root growth, indicating that xylem colonization and subsequent root inhibition is controlled by other, still-undefined HrpGregulated genes. 
Auxin signaling alterations caused by $R$. solanacearum infection likely trigger root structure rearrangements, resulting in root-hair formation.

Our plant mutant analysis showed that neither of the defense regulators salicylic acid, jasmonic acid, ethylene, or NADPHproduced ROS were required for any of the root phenotypes observed after $R$. solanacearum GMI1000 infection. On the contrary, we showed that auxin signaling was clearly required for infection-triggered root-hair formation. This is not surprising, since auxin is one of the main orchestrators of root-hair formation (Grierson et al. 2014; Lee and Cho 2013) and can promote this process (Pitts et al. 1998). Root hairs are outgrowths of epidermal cells that contribute to nutrient and water absorption (Grierson et al. 2014), but they also participate in plant-microbe interactions. For instance, root hairs are the entry point of both mutualistic rhizobacteria (Rodríguez-Navarro et al. 2007) and pathogenic bacteria such as Plasmodiophora brassicaceae, the causal agent of clubroot disease (Kageyama and Asano 2009). Interestingly, auxin signaling was proposed to promote cell-wall remodeling to allow root-hair growth (Breakspear et al. 2014) and it has been shown to be a key component of both pathogenic and mutualistic root-hair infections (Jahn et al. 2013; Laplaze et al. 2015).

During $R$. solanacearum-Arabidopsis interactions, auxin signaling may have additional important roles beyond its involvement in root-hair formation. $R$. solanacearum inoculation resulted in an induction of DR5rev::GFP expression in the root vascular cylinder at early stages of infection, indicative of increased auxin signaling levels. Furthermore, plant infection results in increased expression of several auxin-related genes (Zuluaga et al. 2015). On a hypothetic scenario, $R$. solanacearum could directly and specifically (e.g., via a T3SS effector) manipulate one or more of the host auxin signaling pathways to its own benefit. There are many examples of effector-mediated manipulation of the host auxin pathway (Kazan and Lyons 2014). In most cases the pathogen uses its type III effector
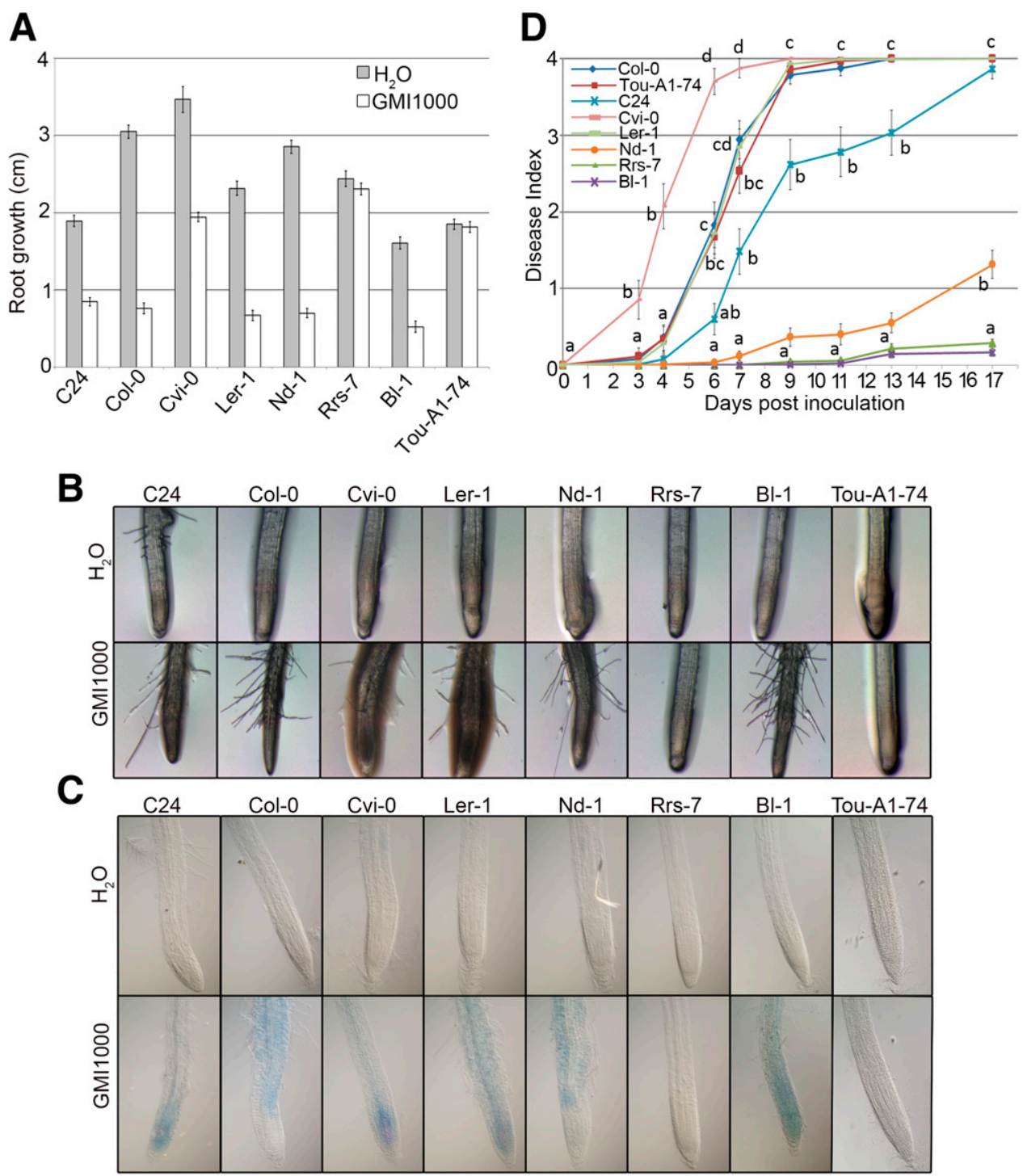

Fig. 6. The absence of the triple phenotype caused by Ralstonia solanacearum in Arabidopsis is indicative of resistance. Six-day-old Arabidopsis seedlings from ecotypes C24, Col-0, Cvi-0, Ler-1, Nd-1, Rrs-7, Bl-1, and Tou-A1-74 were inoculated with R. solanacearum GMI1000 or water and, at 6 days postinoculation, A, root growth was measured, B, root hair was visualized, and $\mathbf{C}$, cell death was observed after Evans blue staining. D, Five-week old plants grown in Jiffy pots were inoculated with GMI1000. The disease index measured symptoms on a 1 to 4 scale $(0=$ no wilting, $1=25 \%$ wilted leaves, $2=50 \%$, $3=75 \%$, and $4=$ death). Letters indicate statistical significance; values not sharing letters represent significant mean differences by one-way analysis of variance $(P<0.05, \alpha=0.05)$ with post hoc Scheffé $(\alpha=0.05)$. The statistical test was applied separately for each timepoint. Seven to 13 plants were used in each of three experiments. 
arsenal to specifically increase auxin levels in the host by targeting auxin biosynthesis, signaling, or transport. Elevated auxin levels are beneficial for many pathogens, toward which auxin promotes susceptibility. This is the case of Pseudomonas syringae, Xanthomonas oryzae, and Magnaporte oryzae, among others. In rice, elevated susceptibility has been linked to auxininduced loosening of the protective cell wall, which would facilitate pathogen colonization. Other pathogens increase the host susceptibility by secreting auxin into the host, which, in turn, induces auxin production inside the host's cells and promotes susceptibility (Fu et al. 2011). Our data points toward a potential link to increased auxin levels as a result of invasion, although further work needs to be done to determine whether this is directly correlated with an increase in susceptibility. In this context, it also remains to be clarified whether auxin-mediated root-hair formation during infection facilitates $R$. solanacearum invasion or whether it is a mere consequence of elevated auxin levels in certain root cells. Also, it is not known whether root hairs may constitute favorite entry points for the bacteria.

\section{Absence of the triple-root phenotype to screen \\ for $\boldsymbol{R}$. solanacearum virulence factors or resistance in Arabidopsis.}

When analyzing different $R$. solanacearum strains, the absence of the root phenotypes is directly linked to the inability of the bacterium to cause symptoms. Thus, strains not capable of inducing the triple-root phenotype show low pathogenicity on Arabidopsis, as is the case for NCPPB3987, UY031, and UW551. Presence of the phenotype is not always correlated with increased aggressiveness of a particular strain. CIP301 and CFBP2957 are not pathogenic on Arabidopsis Col-0 plants, despite causing the triple-root phenotype. Gene-for-gene interactions may mask these root phenotypic features and block $R$. solanacearum before it starts causing wilt. This may indicate that the Col-0 accession possesses resistance proteins that recognize effectors secreted by the two phylotype IIA strains or that phylotype IIA strains lack one or several virulence factors required to establish disease on Arabidopsis or repress some plant defenses. Similarly, the $h r p G$ mutant, which has an extremely reduced capacity to invade the xylem, does not cause root inhibition (discussed above).

Our data show that the lack of the triple-root phenotype can be linked to resistance to $R$. solanacearum. This is the case of Arabidopsis accession Rrs-7, which appears completely resistant to $R$. solanacearum GMI1000 and does not display any of the described root phenotypes. Resistance to $R$. solanacearum is very rare among Arabidopsis accessions. The clear enrichment of resistant accessions among those lacking the capacity to cause the triple phenotype indicates that the root phenotypes described here can be used to screen plant varieties in search for resistance. The fact that other resistant accessions present the phenotypes may indicate that they possess alternative forms of resistance or that other factors, including gene-for-gene interactions, override the observed phenotypes. This could be the case of the resistant accession Nd-1, which is able to detect $R$. solanacearum GMI1000 infection through recognition of the effector PopP2 by the resistance protein RRS1-R (Deslandes et al. 2003). This system could, thus, be used to differentiate ecotypes with resistances due to a gene-for-gene recognition $(\mathrm{Nd}-1$ resistance associated to the presence of the triple response) compared with other resistance mechanisms (Rrs-7 resistance associated with absence of the triple-root response). Along this line, Arabidopsis Bl-1, which also does not wilt but shows clear infection, indicated by the appearance of the root phenotypes, may also recognize $R$. solanacearum through an alternative effectorresistance protein pair and stop invasion.
Taken together, our results on both the bacterial and the plant side favor the notion that absence of the root phenotypes is indicative of ineffective colonization that may reflect novel forms of resistance. Thus, the absence of the root phenotypes described here could help in the search for plant varieties with higher resistance to devastating bacterial wilt disease.

\section{MATERIALS AND METHODS}

\section{Biological material.}

Arabidopsis thaliana ecotypes B1-1, C24, Col-0, Cvi-0, Ler-1, Nd-1, Rrs-7 (Clark and Schweikert 2007; Delker et al. 2010), Tou-A1-74 (Horton et al. 2012), and the Col-0 mutants sid2-2, dde2-2, and ein2-1 (Tsuda et al. 2009), tirl-1 (Dharmasiri et al. 2005), tirl-1/afb2-3 (Parry et al. 2009), jarl-1 (Staswick and Tiryaki 2004), jai3-1 (Chini et al. 2007), and atrbohD and atrbohF (Torres et al. 2002) were used. The Col-0 transgenic line DR5rev::GFP (Friml et al. 2003) was used to monitor auxin signaling.

All $R$. solanacearum strains used are described in Supplementary Table $\mathrm{S} 1$. Bacteria were grown at $28^{\circ} \mathrm{C}$ in solid or liquid rich $\mathrm{B}$ medium (1\% Bacto peptone, $0.1 \%$ yeast extract, and $0.1 \%$ casamino acids, all from Becton, Dickinson and Co. [Franklin Lakes, NJ, U.S.A.]), adding the appropriate antibiotics as described by Monteiro et al. (2012).

\section{In vitro inoculation assay.}

Seeds were sterilized, with a solution containing $30 \%$ bleach and $0.02 \%$ Triton-X 100, for $10 \mathrm{~min}$, were washed five times with Milli-Q water, and were sown (20 seeds per plate) on MS plates containing vitamins (Duchefa Biochemie B.V., Haarlem, The Netherlands) and $0.8 \%$ agar (Becton, Dickinson and Co.). Sown plates were stratified at $4{ }^{\circ} \mathrm{C}$ in the dark for 2 days. Then, plates were transferred to chambers and were grown for 6 to 7 days under constant conditions of 21 to $22^{\circ} \mathrm{C}, 60 \%$ humidity, and a 16-h light and 8-h dark photoperiod.

For inoculation, $R$. solanacearum was collected, by centrifugation $(1,300 \times g, 5 \mathrm{~min})$, from overnight liquid cultures, was resuspended with water, and was adjusted to a final optical density at $600 \mathrm{~nm}\left(\mathrm{OD}_{600}\right)$ of 0.01 . Six- to 7-day-old Arabidopsis seedlings, grown on plates as detailed above, were inoculated with $5 \mu$ of the bacterial solution, which was applied $1 \mathrm{~cm}$ above the root tip, as described previously (Digonnet et al. 2012). Plates with the infected seedlings were sealed with micropore tape (3M Deutschland $\mathrm{GmbH}$, Neuss, Germany) and were transferred to a controlled growth chamber at $25^{\circ} \mathrm{C}, 60 \%$ humidity, and a 12-h light and 12-h dark photoperiod. Root length of infected seedlings was recorded over time. For roothair evaluation, pictures were taken 6 dpi with an Olympus DP71 stereomicroscope (Olympus, Center Valley, PA, U.S.A.) at $11.5 \times$. To analyze cell death, roots from seedlings grown on plates were collected 6 dpi and were immediately stained by carefully submerging them into a solution containing $0.05 \%$ (wt/vol) of Evans blue (Sigma-Aldrich, Buchs, Switzerland) for $30 \mathrm{~min}$ at room temperature. Roots were then washed twice with distilled water and were photographed under a $20 \times$ lens with a Nomarski Axiophot DP70 microscope (Zeiss, Oberkochen, Germany). For propidium iodide staining, roots of infected seedlings were soaked into a $1-\mu \mathrm{g} / \mathrm{ml}$ staining solution (Sigma-Aldrich) and were immediately photographed with a $20 \times$ magnification on an Olympus FV1000 (Olympus) or a Leica SP5 (Wetzlar, Germany) confocal microscope.

\section{Pathogenicity assays.}

$R$. solanacearum pathogenicity tests were carried out using the soil-drench method (Monteiro et al. 2012). Briefly, Arabidopsis 
was grown for 4 to 5 weeks on Jiffy pots (Jiffy Group, Lorain, $\mathrm{OH}$, U.S.A.) in a controlled chamber at $22^{\circ} \mathrm{C}, 60 \%$ humidity, and an 8-h light and 16-h dark photoperiod. Jiffys were cut at one-third from the bottom and were immediately submerged for $30 \mathrm{~min}$ into a solution of overnight-grown $R$. solanacearum adjusted to $\mathrm{OD}_{600}=0.1$ with distilled water $(35 \mathrm{ml}$ of bacterial solution per plant). Then, inoculated plants were transferred to trays containing a thin layer of soil drenched with the same $R$. solanacearum solution and were kept in a chamber at $28^{\circ} \mathrm{C}$, $60 \%$ humidity, and $12 \mathrm{~h}$ of light and $12 \mathrm{~h}$ of dark. Plant wilting symptoms were recorded every day and were expressed according to a disease index scale $(0=$ no wilting, $1=25 \%$ wilted leaves, $2=50 \%, 3=75 \%$, and $4=$ death). At least 30 plants were used in each assay, performed in at least three replicate experiments.

$R$. solanacearum vessel colonization was tested in Arabidopsis plants inoculated with a lower inoculum $\left(\mathrm{OD}_{600}=0.01\right)$. To quantify bacterial colonization, the plant aerial parts were cut 14 days after inoculation and were homogenized. Dilutions of the homogenate plant material were plated on rich B medium supplemented with the appropriate antibiotics and the bacterial content was measured as CFU per gram of fresh plant tissue. At least 20 plants were inoculated per $R$. solanacearum strain and the experiment was repeated three times.

\section{ACKNOWLEDGMENTS}

We are grateful to M. Estelle (University of California San Diego) for the tirl and tirl/afb2 seeds; to K. Tsuda (Max Planck Institute for Plant Breeding Research) for the sid2, ein2, and $d d e 2$ seeds; to R. Solano (Spanish National Center for Biotechnolgy) for the jai3-1 and jar1-1 seeds; and to M. Quint (Leibniz institute of Plant Biochemistry) for Arabidopsis accessions B1-1, C24, Cvi-0, Ler-1, Nd-1, and RRS-7. We thank M. A. Moreno-Risueño for helpful comments and critically reading the manuscript. We also thank S. Poussier, I. Robène, E. Wicker, S. Genin, C. P. Cheng, P. Hanson, and P. Prior for providing $R$. solanacearum strains and for their advice in the choice of relevant ones. This work was funded by Ministry of Economy and Competitiveness (MINECO) projects AGL201346898-R and AGL2016-78002-R to N. S. Coll and M. Valls and RyC 2014 1658 to N. S. Coll and EU-Marie Curie Actions (PCDMC-321738 and PIIF-331392) and BP_B 00030 from the Catalan Government to N. S. Coll. We also want to acknowledge the support of the COST Action SUSTAIN (FA1208), the "Severo Ochoa Programme for Centres of Excellence in R\&D" 2016-2019 (SEV-2015-0533) from the MINECO, and by the Centres de Recerca de Catalunya programme and the government of Catalonia.

\section{LITERATURE CITED}

Boucher, C. A., Barberis, P. A., Trigalet, A. P., and Demery, D. A. 1985. Transposon mutagenesis of Pseudomonas solanacearum: Isolation of Tn5-induced avirulent mutants. J. Gen. Microbiol. 131:2449-2457.

Breakspear, A., Liu, C., Roy, S., Stacey, N., Rogers, C., Trick, M., Morieri, G., Mysore, K. S., Wen, J., Oldroyd, G. E. D., Downie, J. A., and Murray, J. D. 2014. The root hair "infectome" of Medicago truncatula uncovers changes in cell cycle genes and reveals a requirement for auxin signaling in rhizobial infection. Plant Cell 26:4680-4701.

Brito, B., Aldon, D., Barberis, P., Boucher, C., and Genin, S. 2002. A signal transfer system through three compartments transduces the plant cell contact-dependent signal controlling $R$. solanacearum hrp genes. Mol. Plant-Microbe Interact. 15:109-119.

Clark, R. M., and Schweikert, G. 2007. Common sequence polymorphisms shaping genetic diversity in Arabidopsis thaliana. Science 317:338-342.

Cruz, A. P., Ferreira, V., Pianzzola, M. J., Siri, M. I., Coll, N. S., and Valls, M. 2014. A novel, sensitive method to evaluate potato germplasm for bacterial wilt resistance using a luminescent Ralstonia solanacearum reporter strain. Mol. Plant-Microbe Interact. 27:277-285.

Curtis, M. J., and Hays, J. B. 2007. Tolerance of dividing cells to replication stress in UVB-irradiated Arabidopsis roots: Requirements for DNA translesion polymerases eta and zeta. DNA Repair (Amst.) 6:1341-1358.

Chini, A., Fonseca, S., Fernandez, G., Adie, B., Chico, J. M., Lorenzo, O. 2007. The JAZ family of repressors is the missing link in jasmonate signalling. Nature 448:666-671.
Delker, C., Poschl, Y., Raschke, A., Ullrich, K., Ettingshausen, S., Hauptmann, V., Grosse, I., and Quint, M. 2010. Natural variation of transcriptional auxin response networks in Arabidopsis thaliana. Plant Cell 22:2184-2200.

Deslandes, L., Olivier, J., Peeters, N., Feng, D. X., Khounlotham, M., Boucher, C., Somssich, I., Genin, S., and Marco, Y. 2003. Physical interaction between RRS1-R, a protein conferring resistance to bacterial wilt, and PopP2, a type III effector targeted to the plant nucleus. Proc. Natl. Acad. Sci. U.S.A. 100:8024-8029.

Deslandes, L., Pileur, F., Liaubet, L., Camut, S., Can, C., Williams, K., Holub, E., Beynon, J., Arlat, M., and Marco, Y. 1998. Genetic characterization of RRS1, a recessive locus in Arabidopsis thaliana that confers resistance to the bacterial soilborne pathogen Ralstonia solanacearum. Mol. Plant-Microbe Interact. 11:659-667.

Dharmasiri, N., Dharmasiri, S., and Estelle, M. 2005. The F-box protein TIR1 is an auxin receptor. Nature 435:441-445.

Digonnet, C., Martinez, Y., Denance, N., Chasseray, M., Dabos, P., Ranocha, P., Marco, Y., Jauneau, A., and Goffner, D. 2012. Deciphering the route of Ralstonia solanacearum colonization in Arabidopsis thaliana roots during a compatible interaction: Focus at the plant cell wall. Planta 236: 1419-1431.

Friml, J., Vieten, A., Sauer, M., Weijers, D., Schwarz, H., Hamann, T. Offringa, R., and Jurgens, G. 2003. Efflux-dependent auxin gradients establish the apical-basal axis of Arabidopsis. Nature 426:147-153.

Fu, J., Liu, H., Li, Y., Yu, H., Li, X., Xiao, J., and Wang, S. 2011. Manipulating broad-spectrum disease resistance by suppressing pathogen-induced auxin accumulation in rice. Plant Physiol. 155:589-602.

Gaff, D. F., and Okong'O-gola, O. 1971. The use of non-permeating pigments for testing the survival of cells. J. Exp. Bot. 22:756-758.

Galán, J. E., and Collmer, A. 1999. Type III secretion machines: Bacterial devices for protein delivery into host cells. Science 284:1322-1328.

Genin, S. 2010. Molecular traits controlling host range and adaptation to plants in Ralstonia solanacearum. New Phytol. 187:920-928.

Genin, S., and Denny, T. P. 2012. Pathogenomics of the Ralstonia solanacearum species complex. Annu. Rev. Phytopathol. 50:67-89.

Grierson, C., Nielsen, E., Ketelaarc, T., and Schiefelbein, J. 2014. Root hairs. Arabidopsis Book 12:e0172.

Hayward, A. C. 1991. Biology and epidemiology of bacterial wilt caused by Pseudomonas solanacearum. Annu. Rev. Phytopathol. 29:65-87.

Hanemian, M., Zhou, B., Deslandes, L., Marco, Y., and Trémousaygue, D. 2013. Hrp mutant bacteria as biocontrol agents: Toward a sustainable approach in the fight against plant pathogenic bacteria. Plant Signal. Behav. 8:e25678.

Horton, M. W., Hancock, A. M., Huang, Y. S., Toomajian, C., Atwell, S., Auton, A., Muliyati, N. W., Platt, A., Sperone, F. G., Vilhjálmsson, B. J., Nordborg, M., Borevitz, J. O., and Bergelson, J. 2012. Genome-wide patterns of genetic variation in worldwide Arabidopsis thaliana accessions from the RegMap panel. Nat. Genet. 44:212-216.

Hueck, C. J. 1998. Type III protein secretion systems in bacterial pathogens of animals and plants. Microbiol. Mol. Biol. Rev. 62:379-433.

Jahn, L., Mucha, S., Bergmann, S., Horn, C., Staswick, P., Steffens, B., Siemens, J., and Ludwig-Müller, J. 2013. The clubroot pathogen (Plasmodiophora brassicae) influences auxin signaling to regulate auxin homeostasis in Arabidopsis. Plants 2:726-749.

Kageyama, K., and Asano, T. 2009. Life cycle of Plasmodiophora brassicae. J. Plant Growth Regul. 28:203-211.

Kazan, K., and Lyons, R. 2014. Intervention of phytohormone pathways by pathogen effectors. Plant Cell 26:2285-2309.

Laplaze, L., Lucas, M., and Champion, A. 2015. Rhizobial root hair infection requires auxin signaling. Trends Plant Sci. 20:332-334.

Lee, R. D., and Cho, H. T. 2013. Auxin, the organizer of the hormonal/ environmental signals for root hair growth. Front. Plant Sci. 4:448.

Lindgren, P. B., Peet, R. C., and Panopoulos, N. J. 1986. Gene cluster of Pseudomonas syringae pv. phaseolicola controls pathogenicity of bean plants and hypersensitivity of nonhost plants. J. Bacteriol. 168 $512-522$

Mansfield, J., Genin, S., Magori, S., Citovsky, V., Sriariyanum, M., Ronald, P. 2012. Top 10 plant pathogenic bacteria in molecular plant pathology. Mol. Plant Pathol. 13:614-629.

Monteiro, F., Sole, M., van Dijk, I., and Valls, M. 2012. A chromosomal insertion toolbox for promoter probing, mutant complementation, and pathogenicity studies in Ralstonia solanacearum. Mol. Plant-Microbe Interact. 25:557-568.

Parry, G., Calderon-Villalobos, L. I., Prigge, M., Peret, B., Dharmasiri, S., Itoh, H., Lechner, E., Gray, W. M., Bennett, M., and Estelle, M. 2009 Complex regulation of the TIR1/AFB family of auxin receptors. Proc. Natl. Acad. Sci. U.S.A. 106:22540-22545. 
Pitts, R. J., Cernac, A., and Estelle, M. 1998. Auxin and ethylene promote root hair elongation in Arabidopsis. Plant J. 16:553-560.

Rodríguez-Navarro, D. N., Dardanelli, M. S., and Ruiz-Sainz, J. E. 2007. Attachment of bacteria to the roots of higher plants. FEMS Microbiol. Lett. 272:127-136.

Saile, E., McGarvey, J. A., Schell, M. A., and Denny, T. P. 1997. Role of extracellular polysaccharide and endoglucanase in root invasion and colonization of tomato plants by Ralstonia solanacearum. Phytopathology 87:1264-1271.

Staswick, P. E., and Tiryaki, I. 2004. The oxylipin signal jasmonic acid is activated by an enzyme that conjugates it to isoleucine in Arabidopsis. Plant Cell 16:2117-2127.

Torres, M. A., Dangl, J. L., and Jones, J. D. 2002. Arabidopsis gp91phox homologues AtrbohD and AtrbohF are required for accumulation of reactive oxygen intermediates in the plant defense response. Proc. Natl. Acad. Sci. U.S.A. 99:517-522.

Tsuda, K., Sato, M., Stoddard, T., Glazebrook, J., and Katagiri, F. 2009. Network properties of robust immunity in plants. PLoS Genet. 5:e1000772.

Turner, M., Jauneau, A., Genin, S., Tavella, M. J., Vailleau, F., Gentzbittel, L., and Jardinaud, M. F. 2009. Dissection of bacterial wilt on Medicago truncatula revealed two type III secretion system effectors acting on root infection process and disease development. Plant Physiol. 150: 1713-1722.
Vailleau, F., Sartorel, E., Jardinaud, M. F., Chardon, F., Genin, S., Huguet, T., Gentzbittel, L., and Petitprez, M. 2007. Characterization of the interaction between the bacterial wilt pathogen Ralstonia solanacearum and the model legume plant Medicago truncatula. Mol. Plant-Microbe Interact. 20:159-167.

Valls, M., Genin, S., and Boucher, C. 2006. Integrated regulation of the type III secretion system and other virulence determinants in Ralstonia solanacearum. PLoS Pathog. 2:e82.

Vasse, J., Frey, P., and Trigalet, A. 1995. Microscopic studies of intercellular infection and protoxylem invasion of tomato roots by Pseudomonas solanacearum. Mol. Plant Microbe Interact. 8:241-251.

Vasse, J., Genin, S., Frey, P., Boucher, C., and Brito, B. 2000. The hrpB and hrpG regulatory genes of Ralstonia solanacearum are required for different stages of the tomato root infection process. Mol. Plant-Microbe Interact. 13:259-267.

Zolobowska, L., and Van Gijsegem, F. 2006. Induction of lateral root structure formation on petunia roots: A novel effect of GMI1000 Ralstonia solanacearum infection impaired in Hrp mutants. Mol. Plant-Microbe Interact. 19:597-606.

Zuluaga, A. P., Sole, M., Lu, H., Gongora-Castillo, E., Vaillancourt, B., Coll, N., Buell, C. R., and Valls, M. 2015. Transcriptome responses to Ralstonia solanacearum infection in the roots of the wild potato Solanum commersonii. BMC Genomics 16:246. 